\title{
Evaluating the Impact of Information Distortion on Normalized Compression Distance
}

\author{
Ana Granados, Manuel Cebrián, David Camacho and Francisco B. Rodríguez \\ Departamento de Ingeniería Informática, Universidad Autonóma de Madrid, Spain, \\ (Ana.GranadosF, Manuel.Cebrian, David.Camacho, F.Rodriguez) @uam.es
}

\begin{abstract}
In this paper we apply different techniques of information distortion on a set of classical books written in English. We study the impact that these distortions have upon the Kolmogorov complexity and the clustering by compression technique (the latter based on Normalized Compression Distance, NCD). We show how to decrease the complexity of the considered books introducing several modifications in them. We measure how the information contained in each book is maintained using a clustering error measure. We find experimentally that the best way to keep the clustering error is by means of modifications in the most frequent words. We explain the details of these information distortions and we compare with other kinds of modifications like random word distortions and unfrequent word distortions. Finally, some phenomenological explanations from the different empirical results that have been carried out are presented.
\end{abstract}

\section{Introduction}

A natural measure of similarity assumes that two objects $x$ and $y$ are similar if the basic blocks of $x$ are in $y$ and vice versa. If this happens we can describe object $x$ by making reference to the blocks belonging to $y$, thus the description of $x$ will be very simple using the description of $y$.

This is what a compressor does to code the concatenated $x y$ sequence: a search for information shared by both sequences in order to reduce the redundancy of the whole sequence. If the result is small, it means that a lot of information contained in $x$ can be used to code $y$, following the similarity conditions described in the previous paragraph. This was formalized by Cilibrasi and Vitányi [1, giving rise to the concept of Normalized Compression Distance (NCD), which is based on the use of compressors to provide a measure of the similarity between the objects. This distance may then be used to cluster those objects.

The mathematical formulation is as follows

$$
N C D(x, y)=\frac{\max \{C(x y)-C(x), C(y x)-C(y)\}}{\max \{C(x), C(y)\}},
$$

where $C$ is a compression algorithm, $C(x)$ is the size of the C-compressed version of $x$, and $C(x y)$ is the compressed size of the concatenation of $x$ and $y$. 
NCD generates a non-negative number $0 \leq N C D(x, y) \leq 1$. Distances near 0 indicate similarity between objects, while distances near 1 reveal dissimilarity.

The theoretical foundations for this measure can be traced back to the notion of Kolmogorov Complexity $K(X)$ of a string $X$, which is the size of the shortest program able to output $X$ in a universal Turing machine [2]3|4]. As this function is incomputable due to the Halting problem [5], the most usual estimation is based on data compression: $C(X)$ is considered a good upper estimate of $K(x)$, assuming that $C$ is a reasonably good compressor for $X[1]$.

In our work we apply this distance to text clustering [16], with the aim to study the way in which the method is influenced by different types of information distortion. A percentage of words of the books is distorted by using two different word-replacing techniques, which eventually change the amount of information remaining in the books, their (estimated) Kolmogorov Complexity, and the clustering error obtained using the NCD.

Other authors [7] have given a theoretical and experimental basis for explaining the NCD-clustering behavior of elements which have been transmitted through a symmetric-channel, i.e. which have been perturbed by a certain amount of uniform random noise. We go a step further by considering a wider spectrum of information distortions, within the framework of a complete experimental setup on a selected text corpus for which an ideal clustering is already known.

The main contributions of this paper are

- New insights for the evaluation and explanation of the behavior of the NCDdriven clustering method,

- a technique to reduce the Kolmogorov complexity of the books while preserving most of the relevant information,

- experimental evidence of how to fine-tune the NCD so that better clustering results are obtained.

This paper is structured as follows. Section 2 describes the distortion/wordreplacement method, the clustering assessment and the Kolmogorov Complexity estimation. Section 3 explains the experimental setup and gathers the results of the experiments. Section 4 summarizes the conclusions and describes ongoing research.

\section{The Distortion Methods}

We want to study the effect of information distortion on NCD-driven text clustering by replacing words from the documents in different manners. After the distortion has been performed, we execute the NCD clustering method on each distorted test set and we quantitatively measure the error of the clustering results obtained. Finally, the Kolmogorov complexity of the distorted documents is estimated, based on the concept that data compression is an upper bound of the Kolmogorov complexity. 


\section{$2.1 \quad$ Replacement methods}

We use six different replacement methods, which are pairwise combinations of two factors: word selection and substitution method.

- Word selection: we incrementally select a percentage $p$, and we eliminate the p-most/least/randomly frequent words in English from the books. We estimate the frequencies of words in English using the British National Corpus [8.

- Substitution method: each character of the word that is distorted according to the word-frequency, is substituted by either a random character or an asterisk.

Note that all six combinations maintain the length of the document. This is enforced to ease the comparison of the Kolmogorov Complexity among several methods.

\section{$2.2 \quad$ Assessing the Clustering}

The CompLearn Toolkit [6] implements the clustering method described in [1]. The clustering method comprises two phases. First, the NCD matrix is calculated using the LZMAX compressor, a Lempel-Ziv-Markov chain Algorithm [9]. Second, the NCD matrix is used as input to the clustering phase and a dendrogram is generated as an output. A dendrogram is an undirected binary tree diagram, frequently used for hierarchical clustering, that illustrates the arrangement of the clusters produced by a clustering algorithm.

In Fig 1 we can observe one of the dendrograms that we have obtained. Each leaf of the dendrogram corresponds to a document, and has a label that starts with the acronym of the author, and ends with the acronym of the title. For example, the node with label AP.AEoM corresponds to the document $A n$ Essay on Man by Alexander Pope.

Once the CompLearn Toolkit is used to cluster the documents, we need to quantitatively evaluate the error of the dendrograms obtained. We define the distance between two nodes as the minimum number of internal nodes needed to go from one to the other. For example, in Fig 1 the distance between the nodes with label $W S . H$ and $W S . A a C$ would be one, since both nodes are connected to the same internal node. We use this concept to measure the error of a dendrogram.

We add all the pairwise distances between nodes starting with the same string, i.e. we add all the pairwise distances between the documents by the same author. For example, in Fig 1 there are three nodes which label starts with $A P$, thus we add the distance between $A P . A E o C$ and $A P . A E o M$, between AP.AEoC and AP.TRotLaOP, and between AP.AEoM and AP.TRotLaOP. We repeat this procedure with the nodes of each author, obtaining a certain total quantity. The bigger the measure, the worse the clustering.

The ideal dendrogram would be a clustering where all the documents by the same author are grouped together. The clustering error corresponding to the ideal dendrogram is 14 for these documents. Note that in Fig 1 the node with 


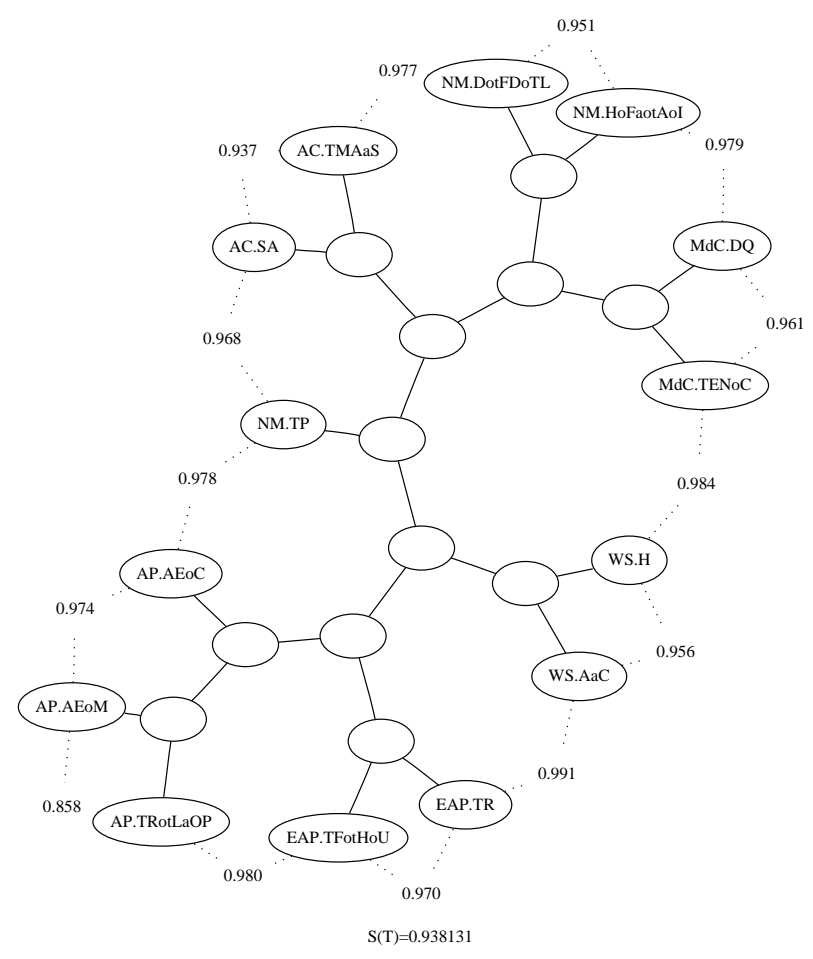

Fig. 1. Example of dendrogram.

label NM.TP is clustered incorrectly. Thus, the clustering error corresponding to this dendrogram is 18 instead of 14 .

\section{Experiments and Results}

The experiments have been designed to evaluate the impact of information distortion on NCD-driven text clustering by incrementally replacing words from the documents in different manners. We measure the error of the clustering in presence of distortion and compare it with two baselines: the ideal clustering, and the non-distorted NCD-driven clustering.

We have applied the NCD clustering method over a set of fourteen classical books written in English. We have two books by Agatha Christie: The Secret Adversary, and The Mysterious Affair at Styles. Three books by Alexander Pope: An Essay on Criticism, An Essay on Man, and The Rape of the Lock, an heroiccomical Poem. Two books by Edgar Allan Poe: The Fall of the House of Usher, and The Raven. Two books by Miguel de Cervantes: Don Quixote, and The Exemplary Novels. Three books by Niccolò Machiavelli: Discourses on the First Decade of Titus Livius, History of Florence and of the Affairs of Italy, and The Prince. Two books by William Shakespeare: The tragedy of Antony and Cleopatra, and Hamlet. 
We show the results of clustering the classical books when the six different replacement methods are used to preprocess them. Every figure plots the clustering error or the complexity of the books vs. a certain percentage of replaced words. The curve with asterisk markers represents the results obtained when the characters of the words are replaced with asterisks. The curve with square markers corresponds to the results obtained when random characters are used to replace the characters of the distorted words. Furthermore, two constant lines may appear in each figure. One corresponds to the measure in the ideal clustering and the other corresponds to the non-distorted NCD-driven clustering. The former is 14 , the latter is 18 .

In every graph, the value on the horizontal axis corresponds to the fraction of the total BNC frequency that is associated to the words being distorted. Note that even when all the words included in the BNC are replaced from the texts, the words that are not included in the BNC remain in the documents. For example, in the book Don Quixote by Miguel de Cervantes, words like Dulcinea or Micomicona, the names of two characters, remain in the documents when all the words of the BNC are distorted from the documents.

The clustering error vs. the percentage of replaced words is presented in Figs 2 1 4 and 6. which show the results for the $X \%$-most/randomly/least frequent words respectively. Figs 3 , 5 and 7 show the evolution of the complexity of the documents as a function of the same percentages.

In Fig 2 we observe that when the characters of the words are replaced with random characters the clustering error increases. When the characters are replaced with asterisks the clustering error remains stable. If we observe the curve with asterisk markers at $80 \%$ and $90 \%$, we can see that the results are better than those obtained for the non-distorted documents, although they are not as good as those that would correspond to the ideal clustering.

Looking at Fig 3 we realize that the complexity of the documents rises when the substitution method is based on random characters. However, when it is based on asterisks the complexity of the documents decreases, because a great amount of characters from the documents are replaced with the same character, which increases the redundancy of the document and thus makes it more compressible.

Fig 4 shows the mean and the standard deviation of the results obtained in ten different experiments. The clustering error increases when random character substitution is applied. However, when asterisk substitution is applied the error keeps stable until $60 \%$. From $60 \%$ to $100 \%$ the error increases. Comparing Figs 2 and 4 we observe that better results are obtained when we start disturbing the most frequent words. This makes us think that the frequency of the replaced words could affect the clustering.

The mean and the standard deviation of the complexity are presented in Fig 5. although the standard deviation is difficult to visualize due to the fact that its absolute value is very small as compared to the mean. Note that this graph decreases faster than the graph which represents the complexity when we start replacing the most frequent words, see Fig 3 . 


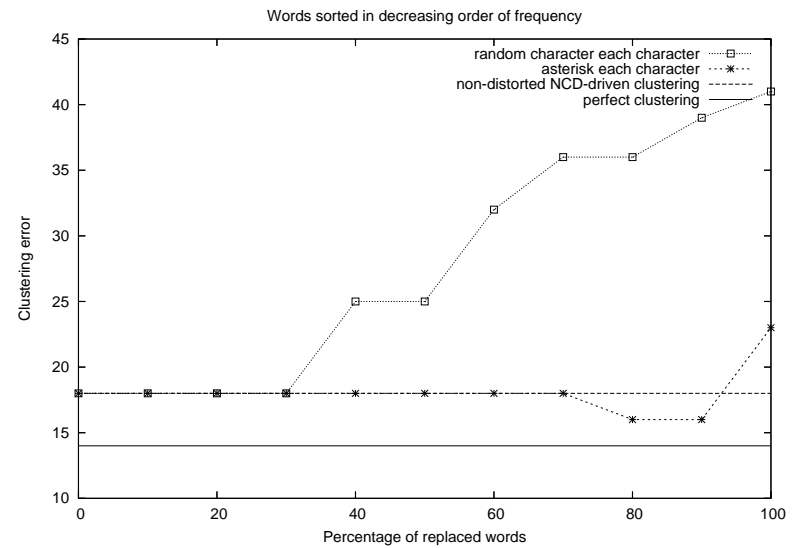

Fig. 2. Clustering error. Words sorted in decreasing order of frequency.

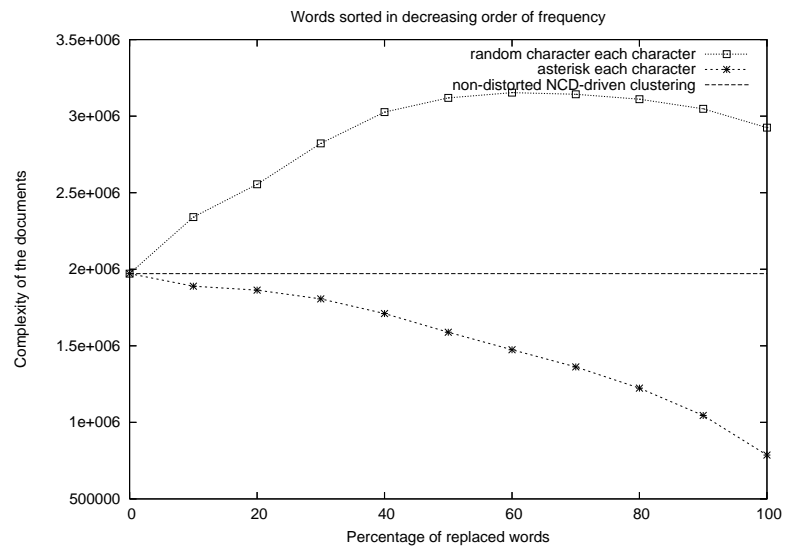

Fig. 3. Complexity of the documents. Words sorted in decreasing order of frequency.

When the characters of the words are replaced with random characters, as shown in Fig 6, the clustering error increases faster than before, see Figs 2 and 4 , When the words are replaced with asterisks the clustering error increases rapidly and then remains stable. This phenomenon could be due to the fact that when we start replacing the least frequent words, we replace precisely those words which carry the most information in terms of clustering compression.

When the substitution method is based on random characters, as shown in Fig 7, the complexity of the documents grows sharply compared to the evolution observed in Figs 3 and 5 . When the substitution method is based on asterisks the complexity of the documents decreases sharply as compared to the same figures. This is due to the fact that when we start disturbing the $\mathrm{X} \%$-least frequent words, lots of words are required to achieve the $10 \%$ of the BNC frequencies.

In order to give a better comparison we illustrate in Fig 8 the clustering error obtained when the words are replaced with asterisks for the three different word 


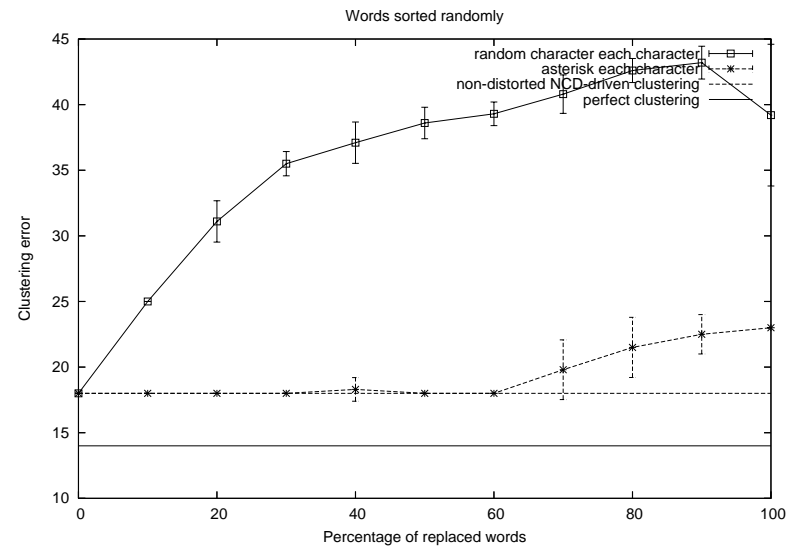

Fig. 4. Clustering error. Words sorted randomly (mean and standard deviation.)

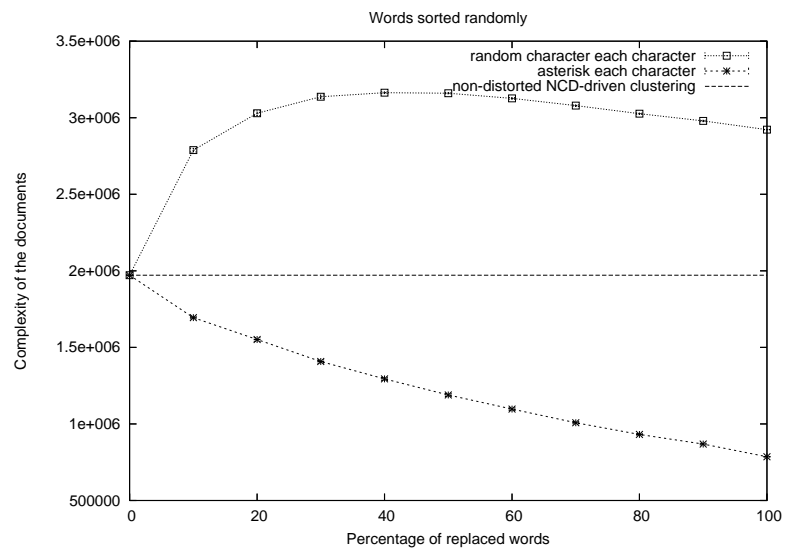

Fig. 5. Complexity of the documents. Words sorted randomly (mean and standard deviation.)

selections: p-most/least/randomly frequent words in English. We observe that the better results are obtained when we start distorting the $p$-most frequent ones, and the worst results are obtained then we start distorting the $p$-least frequent ones. When we select randomly the words the results keep between the others. These facts empirically demonstrate that the frequency of the words affects the clustering results when we cluster these books using the CompLearn Tool with the LZMAX compressor.

In an analogous way, the complexity of the documents for the three word selection techniques is depicted in Fig 9. Comparing Figs 8 and 9 we observe that document complexity and clustering error are negatively correlated. Thus, the best clustering results are obtained using the word selection that reduces the least the complexity of the documents. 


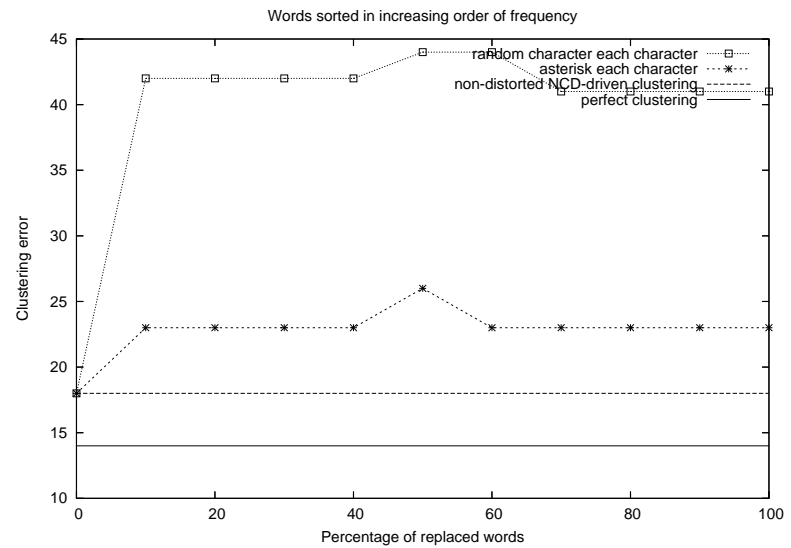

Fig. 6. Clustering error. Words sorted in increasing order of frequency.

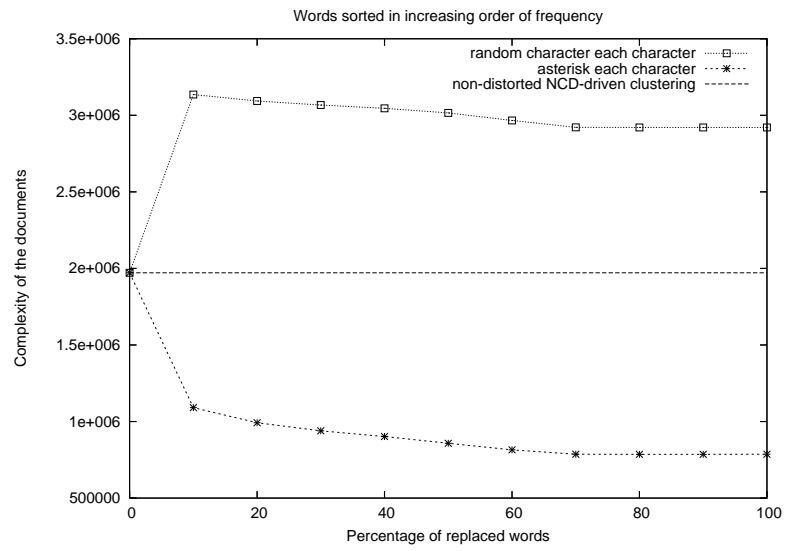

Fig. 7. Complexity of the documents. Words sorted in increasing order of frequency.

To summarize, when random characters are used to replace the words in the text preprocessing phase, the error of the clustering method increases with the percentage of words removed, independently of the word selection used. When the words are replaced using asterisks the clustering error is always smaller than the one obtained when using random characters. Furthermore, the best clustering results are obtained when we select the most frequent words, and the substitution method is based on asterisks. In this case, for $80 \%$ and $90 \%$, the results obtained from the original texts are improved. Moreover, comparing all the figures, it can be observed that the frequency of the removed words has an influence over the clustering error. 


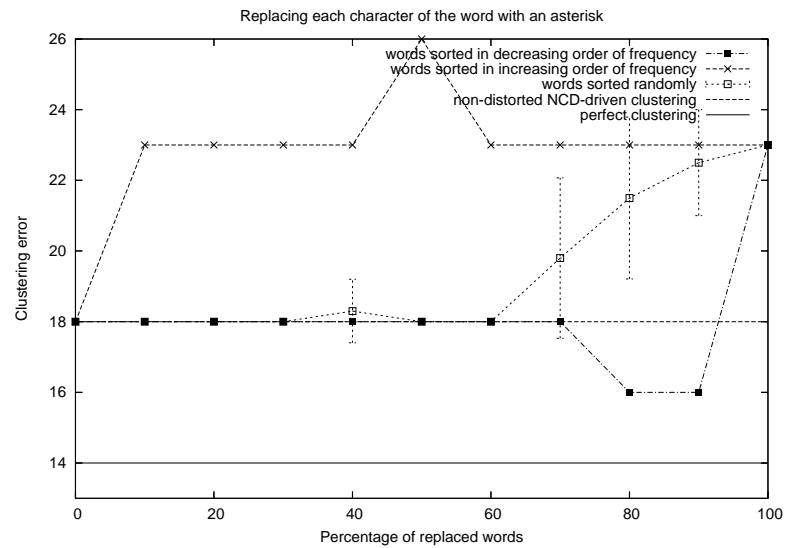

Fig. 8. Clustering Results. Asterisk each character of the replaced word.

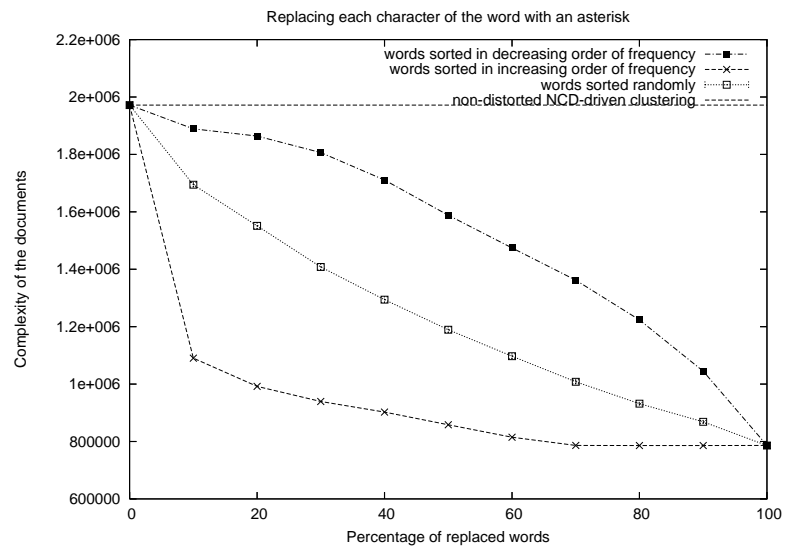

Fig. 9. Complexity of the documents. Asterisk each character of the replaced word.

\section{Conclusions and Discussion}

We have applied the clustering method detailed in [1] to cluster several English classical books. We have studied how the clustering method is affected by different types of information distortion. In order to do that, we have measured the clustering error vs. the percentage of words distorted. The Kolmogorov complexity of the books has been estimated as well, to study the impact of the information distortion on the complexity of the documents.

Although several distortion methods have been designed, in this paper we have only considered those which maintain the initial length of the books to ease the comparison of the Kolmogorov complexity among them.

Three main contributions have been presented in this paper. First, we have made an empirical evaluation of the behavior of the NCD-driven clustering method, and the way in which an incremental modification of the books af- 
fects the clustering error. Second, we have presented a technique which reduces the Kolmogorov complexity of the books while preserving the relevant information therein. Third, we have observed experimental evidence of how to improve the NCD-clustering method by preprocessing the books in a certain manner.

The experimental results show how the clustering error is maintained even when the information contained in the documents is reduced progressively by replacing the words using a special character. We have found that replacing the most frequent words gives us the better clustering results. This method maintains the clustering error with very high values of distortion, and even improves the non-distorted NCD-driven clustering when the $80 \%-90 \%$ of the words are replaced from the documents, see Fig 2 . This means that we are replacing exactly non-relevant parts of the books. This makes it easier for the compressor to estimate the complexity of the documents in an accurately manner. Therefore, the compressor obtains more reliable similarities.

Other techniques, such as randomly selecting the words to replace, or replacing the least frequent ones have been studied and analyzed. Despite the complexity of the documents being reduced too (see Figs 5, and 7), the clustering error increases faster (see Figs 4, and 6). Thus, the information that has been replaced is relevant in the clustering process, and consequently we are losing important information. Therefore, the similarities among the documents are not being correctly measured.

In the future, we plan to work in several ways to study the observed behavior in other textual repositories, like scientific documentation, or genome-based repositories. However, the NCD-based clustering is a general technique so it is possible to use other kinds of sources, such as, music or video. In these domains it would be necessary to analyze how the distortion method could be designed. Other well-known compression algorithms, like PPMZ, BZIP2 or GZIP, will be analyzed to evaluate if the complexity estimation affects the clustering behavior as much as it does in other NCD-driven experiments 10. Finally, we will apply these techniques in other areas like Information Retrieval.

\section{Acknowledgment}

This work was supported by TIN 2004-04363-CO03-03, TIN 2007-65989, CAM S-SEM-0255-2006, TIN2007-64718 and TSI 2005-08255-C07-06. We would also like to thank Franscico Sánchez for his useful comments on this draft.

\section{References}

1. Cilibrasi, R., Vitanyi, P.: Clustering by compression. IEEE Transactions on Information Theory 51(4) (2005) 1523-1545

2. Turing, A.: On computable numbers, with an application to the entscheidungsproblem. Proceedings of the London Mathematical Society 2(42) (1936) 230-265

3. Kolmogorov, A.: Three approaches to the quantitative definition of information. Problems Information Transmission 1(1) (1965) 1-7

4. Li, M., Vitányi, P.: An introduction to Kolmogorov complexity and its applications. SpringerVerlag Graduate Texts In Computer Science Series (1997) 637 
5. Sipser, M.: Introduction to the Theory of Computation. Second edn. PWS Publishing (2006)

6. Cilibrasi, R., Cruz, A.L., de Rooij, S., Keijzer, M.: CompLearn Toolkit. [Online] Available: http://www.complearn.org/

7. Cebrián, M., Alfonseca, M., Ortega, A.: The normalized compression distance is resistant to noise. IEEE Transactions on Information Theory 53(5) (2007) 1895-1900

8. Consortium, B.N.C.: British National Corpus. Oxford University Computing Services [Online] Available: http://www.natcorp.ox.ac.uk/

9. Pavlov, I.: LZMA. [Online] Available: http://www.7-zip.org/sdk.html

10. Cebrián, M., Alfonseca, M., Ortega, A.: Common pitfalls using normalized compression distance: what to watch out for in a compressor. Communications in Information and Systems 5(4) (2005) 367-384 